\title{
BMJ Open Identifying and understanding the health and social care needs of older adults with multiple chronic conditions and their caregivers: a protocol for a scoping review
}

\author{
Elana Commisso, ${ }^{1}$ Katherine S McGilton, ${ }^{1,2}$ Ana Patricia Ayala, ${ }^{3}$ \\ Melissa, K Andrew, ${ }^{4}$ Howard Bergman, ${ }^{5}$ Line Beaudet, ${ }^{6,7}$ Veronique Dubé, ${ }^{7}$ \\ Mikaela Gray, ${ }^{1}$ Lori Hale, ${ }^{8}$ Margaret Keatings, ${ }^{9}$ Emily Gard Marshall, ${ }^{10}$ \\ Janet McElhaney, ${ }^{11,12}$ Debra Morgan, ${ }^{13}$ Edna Parrott, ${ }^{14}$ Jenny Ploeg, ${ }^{15}$ \\ Tara Sampalli, ${ }^{16}$ Douglas Stephens, ${ }^{17}$ Isabelle Vedel, ${ }^{5}$ Jennifer Walker, ${ }^{18}$ \\ Walter P Wodchis, ${ }^{1,19,20}$ Martine T E Puts ${ }^{2}$
}

To cite: Commisso $\mathrm{E}$, McGilton KS, Ayala AP, et al. Identifying and understanding the health and social care needs of older adults with multiple chronic conditions and their caregivers: a protocol for a scoping review. BMJ Open 2017;7:e018247. doi:10.1136/ bmjopen-2017-018247

- Prepublication history and additional material for this paper are available online. To view these files, please visit the journal online (http://dx.doi. org/10.1136/bmjopen-2017018247).

Received 14 June 2017 Revised 10 0ctober 2017 Accepted 15 November 2017

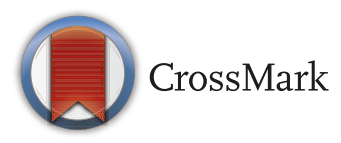

For numbered affiliations see end of article.

Correspondence to Dr Martine T E Puts; martine.puts@utoronto.ca

\section{ABSTRACT}

Introduction People are living longer; however, they are not necessarily experiencing good health and wellbeing as they age. Many older adults live with multiple chronic conditions (MCC), and complex health issues, which adversely affect their day-to-day functioning and overall quality of life. As a result, they frequently rely on the support of friend and/or family caregivers. Caregivers of older adults with MCC often face challenges to their own well-being and also require support. Currently, not enough is known about the health and social care needs of older adults with MCC and the needs of their caregivers or how best to identify and meet these needs. This study will examine and synthesise the literature on the needs of older adults with MCC and those of their caregivers, and identify gaps in evidence and directions for further research.

\section{Methods and analysis We will conduct a scoping} review of the peer-reviewed and grey literature using the updated Arksey and 0'Malley framework. The literature will be identified using a multidatabase and grey literature search strategy developed by a health sciences librarian. Papers, reports and other materials addressing the health and social care needs of older adults and their friend/ family caregivers will be included. Search results will be screened, independently, by two reviewers, and data will be abstracted from included literature and charted in duplicate.

Ethics and dissemination This scoping review does not require ethics approval. We anticipate that study findings will inform novel strategies for identifying and ascertaining the health and social care needs of older adults living with MCC and those of their caregivers. Working with knowledge-user members of our team, we will prepare materials and presentations to disseminate findings to relevant stakeholder and end-user groups at local, national and international levels. We will also publish our findings in a peer-reviewed journal.
Strengths and limitations of this study

This is the first scoping review to identify the care needs and preferences of older adults living with multiple chronic conditions and those of their caregivers, as well as approaches to ascertaining these needs and preferences.

- Additional strengths of this study include the use of established scoping review methods, a systematic search strategy developed by a health sciences librarian, and a systematic approach to screening and data abstraction carried out independently by at least two individuals.

- A further strength of this study is that it will include a broad range of grey literature sources, including government and non-governmental websites and documents.

- A multidisciplinary research team including older adults and caregivers of older adults as well as representatives from stakeholder and decisionmaker groups will be consulted on and actively participate in the study review process.

- Limitations of this review include the language limitations in the search.

\section{INTRODUCTION}

People are living longer; however, they are not necessarily experiencing good health and well-being as they age. ${ }^{1-3}$ Older adults are at a significant risk of having multiple chronic conditions (MCC), also known as multimorbidity, and associated functional impairment. ${ }^{4-7}$ As they continue to age, many older adults live with a growing number of complex health issues that adversely affect their day-to-day functioning and overall 
quality of life (QoL). ${ }^{389}$ For some individuals, and groups of older adults, these issues are further compounded by factors related to social and structural determinants of health. ${ }^{1011}$ This is especially true for older women, ethnocultural minorities, indigenous persons, persons with cognitive impairment and persons with lower socioeconomic status, or living in rural or remote areas. ${ }^{12-15}$

Data demonstrate that being able to age at home, or in an environment of one's choice in the community, and maintaining functional independence are key priorities for older adults. ${ }^{3}{ }^{36-19}$ For persons with MCC, living with complex health issues that hinder their day-to-day functioning, ageing at home can be significantly more challenging and these individuals frequently rely on the support of friend and/or family caregivers to complete activities of daily living. ${ }^{40-22}$ Caregivers of older adults with MCC often face challenges to their own financial, emotional and psychological well-being, and also require social and medical support. ${ }^{23} 24$

In the recently released World Report on Ageing and Health, the WHO called for the development of health systems 'that can ensure affordable access to integrated services that are centred on the needs of older people'. The report acknowledges that older adults' experiences of ageing can vary widely and that diverse individuals and groups of older adults with MCC and their caregivers have unique health and social care needs. ${ }^{3}$ Encouragingly, efforts to align health systems to be more responsive to the needs of ageing populations are underway internationally. ${ }^{382526}$ Currently, however, not enough is known about the health and social care needs of older adults with MCC and those of their friend and family caregivers, including how best to identify, understand and meet these needs.

To date, there are syntheses of existing evidence on the epidemiology of multimorbidity, interventions for persons with MCC and instruments used by physicians to determine care priorities of patients living with MCC. However, to our knowledge, there is no review of the evidence on the health and social care needs of community-dwelling older adults with MCC and their caregivers, or how best to ascertain those needs across diverse populations, and care and living settings. ${ }^{27-30}$ Our project aims to redress this knowledge gap.

In this paper, we set out the protocol for a scoping review to examine the literature on the health and social care needs of community-dwelling older adults with MCC and their family caregivers. The review seeks to answer three research questions:

1. What are the health and social care needs, priorities and preferences of community-dwelling older adults with MCC and those of their friend and family caregivers?

2. How do social and structural determinants of health, such as gender, socioeconomic status, ethnicity and education, impact these needs?

3. What (research) approaches have been used to ascertain these needs and preferences?
The objectives of the review are to synthesise the research and grey literature on the needs of older adults living with MCC and their caregivers and how best to ascertain those needs, to identify gaps in evidence in this area and to set directions for further research. Findings from this review will help guide a research programme with the aim of (1) implementing and testing patient-oriented approaches to identifying and ascertaining the needs of diverse individuals and groups of older adults with MCC and their caregivers and (2) developing function-focused interventions and associated outcome measures informed by patient, caregiver and community needs and preferences.

\section{METHODS AND ANALYSIS}

We will conduct a scoping review to explore the needs experienced by older adults with MCC, and their caregivers, approaches to ascertaining these needs as well as the diverse determinants of health that impact these needs. Our study team includes older adults and family caregivers to help keep the perspectives and experiences of persons living with MCC or caring for them at the forefront of this work. Our team also includes knowledge users (KU) and representatives of other relevant stakeholder groups, such as older adults, their caregivers, clinicians and health-system decision makers, to help facilitate eventual dissemination of our findings.

Scoping review methodology is intended for exploratory research and will allow us to include a wide range of evidence in our synthesis. This feature of the approach and its methodology are particularly pertinent, as our topic, the health and social care needs of older adults with MCC and their caregivers, has been examined using various research approaches (qualitative, quantitative, arts-based and mixed methods approaches) as well as grey literature, some of which are excluded from other types of reviews. Scoping review methods will also permit us to offer a systematic overview of the existing evidence, identify key themes and conduct types of analyses (eg, frequency, thematic, narrative) necessary to explore our research questions methodically and rigorously. Furthermore, as scoping review methodology allows for an iterative approach to refining research questions, the search strategy, the abstract review process and data abstraction, we will continue to engage the full spectrum of our team (including patients, caregivers, and other stakeholders and end users) throughout the research process. $^{31}$

We will use the scoping review methods framework outlined by Arksey and O'Malley and refined by Levac et al, Colquhoun et al and Daudt et al and follow the Preferred Reporting Items for Systematic Reviews and Meta-Analyses Protocol (PRISMA-P) statement. ${ }^{31-34}$ The framework includes six steps: (1) identifying the research questions (listed above); (2) identifying relevant literature; (3) study selection; (4) charting the data; (5) collating, summarising and reporting the 
results; (6) consulting with key stakeholders and translating knowledge.

\section{Identifying relevant literature}

We searched OVID Medline (1946 to present, including Epub Ahead of Print, and In Process \& Other Non-Indexed Citations), OVID Embase (1947 to present), OVID PsycINFO (1806 to present), OVID Social Work Abstracts (1968 to present), EBSCO CINAHL Plus with Full Text (1981 to present), EBSCO AgeLine (1966 to present) and Cochrane Central to identify research articles addressing the subjects of health and social care needs and priorities, and multimorbidity in adults 55 years of age and over. An academic health sciences librarian (APA) developed the search strategies with input from team members (MP, KM, MG and EC). JW consulted on development of search strategies to capture indigenous focused studies. The search strategies were translated using each database platform's command language, controlled vocabulary and appropriate search fields. MeSH terms, EMTREE terms, APA thesauri terms, CINAHL headings and text words were used for the search concepts of health and social care needs and priorities, indigenous populations and multimorbidity. Examples of search terms for the broad concept of care needs, preferences and priorities include health priorities, health services needs and demand, and patient preferences. Examples of terms for the concept indigenous populations include aboriginal, indigenous, native and tribe. Examples of terms for the concept of multimorbidity include comorbidity, MCC, multimorbidity and polypathology.

We applied a modified adult age filter to the Medline strategy. ${ }^{35}$ This filter was translated and applied to the Embase, PsycINFO, CINAHL and Cochrane Central search strategies. The filters were not validated. Language limits were applied to capture articles in English, French, Dutch and German, in all databases where applicable. Final searches were completed in May 2017 and we anticipate completion of study review processes by April 2018. For full Medline strategy, see online supplementary appendix A. Additionally, we will search reference lists of included studies.

We will also conduct a thorough search of grey literature (ie, non-research studies) to identify any non-indexed literature or alternative media, including government, community and policy reports, conference proceedings, practice guidelines and educational materials by searching sources such as OpenGrey, Conference Proceedings Index, ProQuest Dissertations and Theses, as well as Google. In addition to calling on the expertise and experience of team members, we will reach out to experts in fields related to care of older adults with complex health issues in order to facilitate identification of relevant data. Searches have been restricted to recent studies (January 2000 to May 2017) to ensure that data are relevant to current social and health system organisations, and care practices. Covidence systematic review software will be used to facilitate the review. ${ }^{36}$

\section{Study selection}

Studies will be selected through a two-step process. First, two members of our study team will independently review each of the titles and corresponding abstracts against the selection criteria below. Then, two reviewers will retrieve and assess all potentially relevant full-text articles against the selection criteria for inclusion. Team members who have experience in indigenous health research will review articles that focus on indigenous populations. All reviewers will use pilot-tested screening and data abstraction forms developed for this review. In case of disagreement between reviewers, other team members will be called on to come to a consensus. Following the recommendations of Levac et $a l^{31}$ and Daudt $e t a l^{84}$ we will use a systematic review approach to study selection to enhance rigour of the review.

\section{Inclusion criteria}

All published and unpublished literature reporting any quantitative, qualitative, arts-based, mixed or multimethods research, including comparative and non-comparative methods, as well as educational materials, policies and reports related to the health and social care needs of community-dwelling older adults with MCC, and their caregivers will be included. In light of the fact that indigenous persons experience multiple and complex health conditions at younger ages than other populations, we will include all literature that focuses on persons 55 years of age or older living with MCC, in order to capture relevant literature related to the care needs of ageing indigenous persons and indigenous communities. ${ }^{37-39}$ Literature reporting on a wider age range of persons living with MCC that include a subgroup analysis for persons 55 years or older will also be included. We will include all studies that meet the following criteria:

1. report health and/orsocial care needs and/or preferences and priorities of older adults living with MCC

2. report health and/or social care needs of friend and/ or family caregivers of older adults living with MCC

3. detail approaches used to solicit and/or ascertain needs of older adults with MCC and their caregivers.

\section{Exclusion criteria}

Expert opinions, editorials and materials that do not include original data, including systematic reviews, will be excluded as well as studies that focus on older persons living with a single chronic condition.

\section{Data extraction}

All studies included will be reviewed and charted by two reviewers independently using Covidence. The data abstraction form will be piloted using five studies to ensure consistency. Any changes that are necessary will be made in consultation with the team prior to continuing abstraction of remaining articles. When available, data to be extracted will include (but may change based on included studies) study citation, publication type (eg, published, unpublished), study type (eg, quantitative, 
qualitative, report), study characteristics (study setting), patient and/or caregiver characteristics (age, gender, ethnicity, location, number and types chronic conditions), involvement of caregiver, health and social care needs, preferences and challenges identified, and (if categorised) categories/themes used. We will explore barriers and facilitators to ascertaining and identifying needs and if considered in the literature the impact and interplay of social determinants of health on needs and care preferences will also be noted. Further data to be abstracted include approaches used to solicit and/or present information from older adults and their families.

\section{Quality assessment}

The quality of the research studies will be assessed as recommended by Levac et $a l^{31}$ using the Mixed Methods Appraisal Tool (MMAT). ${ }^{40}$ The MMAT allows inclusion of studies using diverse research designs, with quality criteria relevant to each particular study design. It will permit us to summarise the quality of the available research evidence and will be beneficial for identifying and developing future research priorities. No study will be excluded based on the MMAT score alone. To assess alignment with established standards in indigenous health research methods, additional quality criteria will be applied to indigenous-focused studies: (1) indigenous community engagement and (2) integration of indigenous perspectives. The community engagement criterion will be met if the author list includes co-authors from indigenous communities or organisations, or if there is explicit description of community engagement approaches. The indigenous perspective criterion will be met if the research team included (or consulted with) indigenous elders and/or knowledge keepers; established an indigenous advisory structure; used an indigenous theoretical framework; or employed indigenous research methods. ${ }^{41} 42$

\section{Synthesis and presentation of results}

To present an overview of information retrieved and report the extent and nature of the literature, we will present results using two strategies: (1) a numerical overview of the amount, type and distribution of the included literature; (2) a narrative synthesis and mapping of the results. ${ }^{31-34}$ In light of the mandate set by the WHO to view 'the diverse needs of older persons as a continuum of functioning', we will use the International Classification of Functioning, Disability and Health (ICF) framework to support mapping the data and to conduct thematic content analysis. ${ }^{43}$ The ICF framework offers a multidimensional, holistic context within which to understand health and social care needs and preferences as related to overall well-being and QoL, as well as the impact of and social and structural determinants of health on overall functioning. Thus, if appropriate we will use deductive thematic analysis on relevant extracted data to map the studies and grey literature onto the domains of functioning outlined in the framework.
We will summarise the approaches used to ascertain needs and explore whether the approach used impacted the nature and extent of information gathered, as well as describe and summarise policies, reports and other grey literature. As described by Arksey and O'Malley ${ }^{32}$ and by Levac et $a l^{\beta 1}$ charting of the data will be an iterative process that depends on the literature found. Analysis will be conducted by investigator members of the team, along with representatives from stakeholder groups and older adults. Formats for presentation of results will be determined based on relevant target audiences and will rely heavily on input from KU, patient and caregiver members of the project team.

\section{Consultation and knowledge translation}

We take an iterative approach to knowledge synthesis, which aligns with scoping review methodology. ${ }^{31}$ Members of our project team represent a broad spectrum of stakeholder and KU groups, including healthcare practitioners and service providers, health system decision and policy makers, and researchers with experience conducting scoping and systematic reviews and expertise in MCC, as well as older adults and their family caregivers. Furthermore, our team members represent diverse Canadian jurisdictions, including the provinces of Nova Scotia, Ontario, Québec and Saskatchewan. Team members from different sectors and jurisdictions were consulted during study design and they will continue to be involved throughout the various stages of this study, including study selection and data extraction through to selection and tailoring of formats for reporting of findings to diverse end-user and stakeholder audiences. KU, patient and stakeholder team members will also bring their experience and perspectives to bear on the analysis component of the synthesis. We anticipate that categories for analysis will be modified iteratively through these processes. Once the data have been analysed, we will hold a workshop to present findings to the whole team to solicit feedback on final content before broader dissemination and end-of-grant knowledge translation (KT) activities.

\section{Ethics and dissemination}

This scoping review does not require ethics approval. End-of-grant KT activities will be facilitated by our KU partner organisations and will include the dissemination of fact sheets, evidence briefs and reports targeted at diverse end-user and stakeholder audiences. Team members JM and JW will facilitate the sharing of findings with indigenous organisations and the provincial and federal partners who focus on health and social services for older indigenous adults. Additionally, results from the review will be presented at national and international conferences in geriatrics, gerontology, health services and policy; and other health and ageing conferences. We will also publish the results in a peer-reviewed journal.

Our syntheses of the literature about the needs and preferences of older adults with MCC, and their caregivers, will help identify gaps in knowledge, define future 
research priorities and inform the development of new frameworks for, and approaches to, assessing and ascertaining needs of older adults with complex health issues, and the needs of their caregivers. If sufficient literature is found, our team will consider whether there is merit in conducting a future systematic review and meta-analysis. We anticipate that dissemination of findings from this work will advance the state of knowledge on this topic, which will directly support health system reform initiatives underway internationally. This research will also be relevant to system administrators and policymakers and help them address barriers to meeting the care needs of older adults, including inequities, as well as leveraging existing resources to be adapted to address patient and caregiver needs effectively and appropriately.

\section{Author affiliations}

${ }^{1}$ Toronto Rehabilitation Institute, University Health Network, Toronto, Ontario, Canada ${ }^{2}$ Lawrence S. Bloomberg Faculty of Nursing, University of Toronto, Toronto, Ontario, Canada

${ }^{3}$ Gerstein Information Science Centre, University of Toronto, Toronto, Ontario,

Canada

${ }^{4}$ Division of Geriatric Medicine, Dalhousie University, Halifax, Nova Scotia, Canada

${ }^{5}$ Department of Family Medicine, McGill University, Montreal, Quebec, Canada

${ }^{6}$ University of Montréal Hospital Research Centre, Montreal, Québec, Canada

${ }^{7}$ Faculty of Nursing, Université de Montréal, Montreal, Québec, Canada

${ }^{8}$ The Change Foundation, Toronto, Ontario, Canada

${ }^{9}$ Patient and family caregiver team member, NA, Toronto, Canada

${ }^{10}$ Department of Family Medicine, Dalhousie University, Halifax, Nova Scotia, Canada

${ }^{11}$ Health Sciences North Research Institute, Sudbury, Ontario, Canada

${ }^{12}$ Northern Ontario School of Medicine, Sudbury, Ontario, Canada

${ }^{13}$ Canadian Centre for Health and Safety in Agriculture, University of Saskatchewan,

Saskatoon, Saskatchewan, Canada

${ }^{14}$ Patient and caregiver team member, NA, Saskatoon, Saskatchewan, Canada

${ }^{15}$ School of Nursing, McMaster University, Hamilton, Ontario, Canada

${ }^{16}$ Nova Scotia Health Authority, Halifax, Nova Scotia, Canada

${ }^{17}$ Patient and caregiver team members, NA, Toronto, Canada

${ }^{18}$ Laurentian University, Sudbury, Ontario, Canada

${ }^{19}$ Institute of Health Policy, Management and Evaluation, University of Toronto,

Toronto, Ontario, Canada

${ }^{20}$ Institute for Clinical Evaluative Sciences, Toronto, Ontario, Canada

Acknowledgements We would like to acknowledge knowledge-user members of our study team: Debra Bell at the Ontario, Ministry of Health and Long-Term Care; Geoff Fernie at the Toronto Rehabilitation Institute in Ontario; Sheena Grimes at Sun Country Health Region in Saskatchewan; Sandra McKay at VHA Home Health Care in Ontario; David McNeil at Health Sciences North in Ontario; and Samir Sinha at Sinai Health Systems in Ontario, who have provided in-kind support for this project and will be involved in subsequent phases of the study.

Contributors EC, KSM and MP conceived the study. All authors contributed towards the protocol design and study plan. APA developed and conducted the initial search with input from EC, KM, MTEP and JW. EC, KSM, MTEP and JW drafted the protocol, with assistance from MG. MA, APA, LB, HB, VD, MG, LH, MK, JM, EGM, $\mathrm{DM}, \mathrm{EP}, \mathrm{JP}, \mathrm{IV}, \mathrm{JW}, \mathrm{TS}, \mathrm{DS}$ and WPW have read and approved the final protocol. KM is the guarantor of the review.

Funding This work was supported by the Canadian Institutes of Health Research (CIHR) SPOR-PIHCI Knowledge Synthesis grant (Funding Reference Number NKS 150581). It was also supported by Health Sciences North; the Nova Scotia Health Authority; the Saskatchewan Health Research Fund and the Toronto Rehabilitation Institute. MTEP is supported by a CIHR New Investigator Award.

\section{Competing interests None declared.}

Provenance and peer review Not commissioned; externally peer reviewed.

Open Access This is an Open Access article distributed in accordance with the Creative Commons Attribution Non Commercial (CC BY-NC 4.0) license, which permits others to distribute, remix, adapt, build upon this work non-commercially, and license their derivative works on different terms, provided the original work is properly cited and the use is non-commercial. See: http://creativecommons.org/ licenses/by-nc/4.0/

(C) Article author(s) (or their employer(s) unless otherwise stated in the text of the article) 2017. All rights reserved. No commercial use is permitted unless otherwise expressly granted.

\section{REFERENCES}

1. Elmslie K. Against the growing burden of disease. Ottawa, Canada: Public Health Agency of Canada.

2. Atun R. Transitioning health systems for multimorbidity. Lancet 2015;386:721-2.

3. World Health Organization. World report on ageing and health. 2015. Luxembourg: World Health Organization, 2015:1-260.

4. Fortin M, Mercer SW, Salisbury C. Introducing multimorbidity. In: ABC of multimorbidity. 2014: 1-25.

5. Marengoni A, Rizzuto D, Wang HX, et al. Patterns of chronic multimorbidity in the elderly population. J Am Geriatr Soc 2009;57:225-30.

6. Salive ME. Multimorbidity in older adults. Epidemiol Rev 2013;35:75-83.

7. Melis R, Marengoni A, Angleman S, et al. Incidence and predictors of multimorbidity in the elderly: a population-based longitudinal study. PLoS One 2014;9:e103120.

8. Goodwin N, Dixon A, Anderson G, et al. Providing integrated care for older people with complex needs: lessons from seven international studies. The King's Fund, 2014.

9. Pefoyo AJ, Bronskill SE, Gruneir A, et al. The increasing burden and complexity of multimorbidity. BMC Public Health 2015;15:415.

10. World Health Organization. A conceptual framework for action on the social determinants of health conceptual framework for action on the social determinants of health, 2010.

11. Northwood M, Ploeg J, Markle-Reid M, et al. Integrative review of the social determinants of health in older adults with multimorbidity. $J$ Adv Nurs 2017. doi: 10.1111/jan.13408. [Epub ahead of print 3 Aug 2017].

12. Guruge S, Birpreet B, Samuels-Dennis JA. Health status and health determinants of older immigrant women in canada: a scoping review. J Aging Res 2015;2015:1-12.

13. Rochon PA, Bronskill SE, Gruneir A, et al. Older Women's Health In: Bierman AS, eds. Project for an Ontario women's health evidencebased report. Toronto, 2011

14. Kuwornu JP, Lix LM, Shooshtari S. Multimorbidity disease clusters in aboriginal and non-aboriginal caucasian populations in Canada. Chronic Dis Inj Can 2014;34:218-25.

15. Brundisini F, Giacomini M, DeJean D, et al. Chronic disease patients' experiences with accessing health care in rural and remote areas: a systematic review and qualitative meta-synthesis. Ont Health Technol Assess Ser 2013;13:33.

16. Donnelly S, O'Brien M, Begley E, et al. I'd prefer to stay at home but I don't have a choice: meeting older people's preference for care: policy, but what about practice? University College Dublin School of Social Policy, Social Work and Social Justice, 2016.

17. Information. ClfH. Health care in Canada: a focus on seniors and aging: CIHI, 2011.

18. Mortimer J, Green M. Briefing: the health and care of older people in England 2015. London: Age UK, 2015. www. ageuk. org. uk/ professional-resources-home/research/reports/care-andsupport/thehealth-and-care-of-older-people-in-england-2015/ (accessed 22 Jun 2016).

19. Upshur R, Kuluski K, Tracy S. OPINION: rethinking health outcomes in the era of multiple concurrent chronic conditions: Healthy Debate, 2014.

20. Laan W, Bleijenberg N, Drubbel I, et al. Factors associated with increasing functional decline in multimorbid independently living older people. Maturitas 2013;75:276-81.

21. Stenholm S, Westerlund $\mathrm{H}$, Head J, et al. Comorbidity and functional trajectories from midlife to old age: the Health and Retirement Study. J Gerontol A Biol Sci Med Sci 2015;70:332-8.

22. Eckerblad J, Theander K, Ekdahl A, et al. To adjust and endure: a qualitative study of symptom burden in older people with multimorbidity. Appl Nurs Res 2015;28:322-7.

23. Duggleby W, Williams A, Ghosh S, et al. Factors influencing changes in health related quality of life of caregivers of persons with multiple chronic conditions. Health Qual Life Outcomes 2016;14:81.

24. Williams A, Sethi B, Duggleby W, et al. A Canadian qualitative study exploring the diversity of the experience of family caregivers of 
older adults with multiple chronic conditions using a social location perspective. Int J Equity Health 2016;15:40.

25. OECD. Health reform: meeting the challenge of aging and multiple morbidities. OECD Publishing, 2011.

26. Chatterji S, Byles J, Cutler D, et al. Health, functioning, and disability in older adults--present status and future implications. Lancet 2015;385:563-75.

27. Lefèvre T, d'Ivernois JF, De Andrade V, et al. What do we mean by multimorbidity? An analysis of the literature on multimorbidity measures, associated factors, and impact on health services organization. Rev Epidemiol Sante Publique 2014;62:305-14.

28. Marengoni A, Angleman S, Melis R, et al. Aging with multimorbidity: a systematic review of the literature. Ageing Res Rev 2011;10:430-9.

29. Smith SM, Soubhi H, Fortin M, et al. Interventions for improving outcomes in patients with multimorbidity in primary care and community settings. Cochrane Database Syst Rev 2012;4:CD006560.

30. Mangin D, Stephen G, Bismah V, et al. Making patient values visible in healthcare: a systematic review of tools to assess patient treatment priorities and preferences in the context of multimorbidity. BMJ Open 2016;6:e010903.

31. Levac D, Colquhoun H, O'Brien KK. Scoping studies: advancing the methodology. Implement Sci 2010;5:69.

32. Arksey H, O'Malley L. Scoping studies: towards a methodological framework. Int J Soc Res Methodol 2005;8:19-32.

33. Colquhoun HL, Levac D, O'Brien KK, et al. Scoping reviews: time for clarity in definition, methods, and reporting. J Clin Epidemiol 2014;67:1291-4.

34. Daudt HM, van Mossel C, Scott SJ. Enhancing the scoping study methodology: a large, inter-professional team's experience with Arksey and O'Malley's framework. BMC Med Res Methodol 2013;13:1.
35. Kastner M, Wilczynski NL, Walker-Dilks C, et al. Age-specific search strategies for Medline. J Med Internet Res 2006;8:e25.

36. Covidence (Systematic Review Software) [program]. Melbourne, Australia.

37. Reading J. The crisis of chronic disease among aboriginal peoples: a challenge for public health, population health and social policy. Victoria BC: Centre for Aboriginal Health Research, University of Victoria, 2009.

38. LoGiudice D. The health of older aboriginal and torres strait Islander peoples. Australas J Ageing 2016;35:82-5.

39. Centre. FNIG. First Nations Regional Health Survey (RHS) 2008/10: National report on adults, youth and children living in first nations communities. Ottawa: First Nations Information Governance Centre, 2012.

40. Pluye P, Robert E, Cargo M, et al. Proposal: a mixed methods appraisal tool for systematic mixed studies reviews. Montreal: McGill University, Department of Family Medicine, 2011. Mixed methods appraisal tool (MMAT) version 2011.

41. CIHR N. SSHRC (Canadian Institutes of Health Research, Natural Sciences and Engineering Research Council of Canada and Social Sciences and Humanities Research Council of Canada). 2010 Tri-council policy statement: ethical conduct for research involving humans. In: Tri-council policy statement: ethical conduct for research involving humans, 2010.

42. Walker J, Scott G, Jacklin K. Alzheimer's disease and related dementia in Indigenous populations: a systematic review of risk factors. Submitted for publication.

43. World Health Organization. Towards a common language for functioning, disability and health. Geneva: World Health Organization, 2002. 